Research article

\title{
An alternative technique for collecting nail specimens for mycological investigations
}

\author{
HKTA Gunasekera ${ }^{1}$, A Nagahawatta ${ }^{1}, \mathrm{~N}$ de Silva ${ }^{1}$ \\ Sri Lankan Journal of Infectious Diseases 2011 Vol.1 (1); 24-26 \\ DOI: http://dx.doi.org/10.4038/sljid.v1i1.3186 \\ Keywords : Onychomycosis, alternative technique, specimen collection
}

\begin{abstract}
An alternative technique for collection of nail samples in the microscopic diagnosis of onychomycoses is discussed. 54 samples were collected using the conventional technique and the alternative method. 31 of the 54 samples were positive, 30 by the test method (96.8\%), and 14 $(48.4 \%)$ by the conventional method showing the usefulness of the proposed method.
\end{abstract}

\section{Introduction}

Collection of an appropriate specimen of high quality is of utmost importance to optimize the laboratory diagnosis of infections. This is especially true in the diagnosis of onychomycosis, where collection of samples from dystrophic and hypertrophied nails is a tedious task. Conventional methods, as described in textbooks, do not yield good specimens from such nails.

Conventional methods of sample collection advocate scraping or clipping the nails and collecting subungual debris from underneath the nail with the help of scalpel blades, nail clippers and banana scalpels. Subsequently, the nails are cut into small pieces to hasten the digestion of keratin by potassium hydroxide $(\mathrm{KOH})^{1}$. This method is effective for nail specimens which are not markedly dystrophic, such as those seen in housewives, children and in persons living in urban areas. However, in persons living in rural areas and villages such as farmers, labourers and agricultural workers, who often present with onychomycosis of the toe nails, the nails are hypertrophic and dystrophic making it very difficult to take a good specimen employing the conventional methods described above. Other researchers have developed techniques such as micro drilling of the nail plate ${ }^{2}$ and curettage combined with drilling techniques ${ }^{3}$ to improve specimen collection in onychomycosis.

\footnotetext{
${ }^{1}$ Department of Microbiology, Faculty of M edicine, Galle.

Address for correspondence : Ajit Nagahawatta Department of Microbiology, Faculty of Medicine, University of Ruhuna, Sri Lanka email: ajitnagahawatte@yahoo.co.uk
} 


\section{Objective}

To develop an in-house, easy method to collect specimens of nails for the laboratory diagnosis of onychomycosis, which would have an acceptable yield of positive results.

\section{Materials and Methods}

A technique was developed to get better nail samples from dystrophic nails by using microtome blades discarded after being used for histopathology sections. The blades were disinfected by dipping them in $70 \%$ alcohol. Holding the blade at an angle of about $30^{\circ}$ to the nail, the nail was gently sliced from the proximal to the distal end to obtain extremely thin nail shavings using the technique shown in Figure 2. These shavings were clarified in $\mathrm{KOH}$ and examined for the presence of fungal hyphae, spores and yeasts by light microscopy.

In order to test the positivity rate of this technique and compare it with the standard technique, 54 nail samples were taken using both methods. The outcome was taken as the presence or absence of fungal hyphae, spores and yeasts, on microscopy, in the specimens examined.

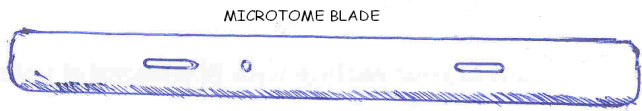

Figure 1-Microtome blade
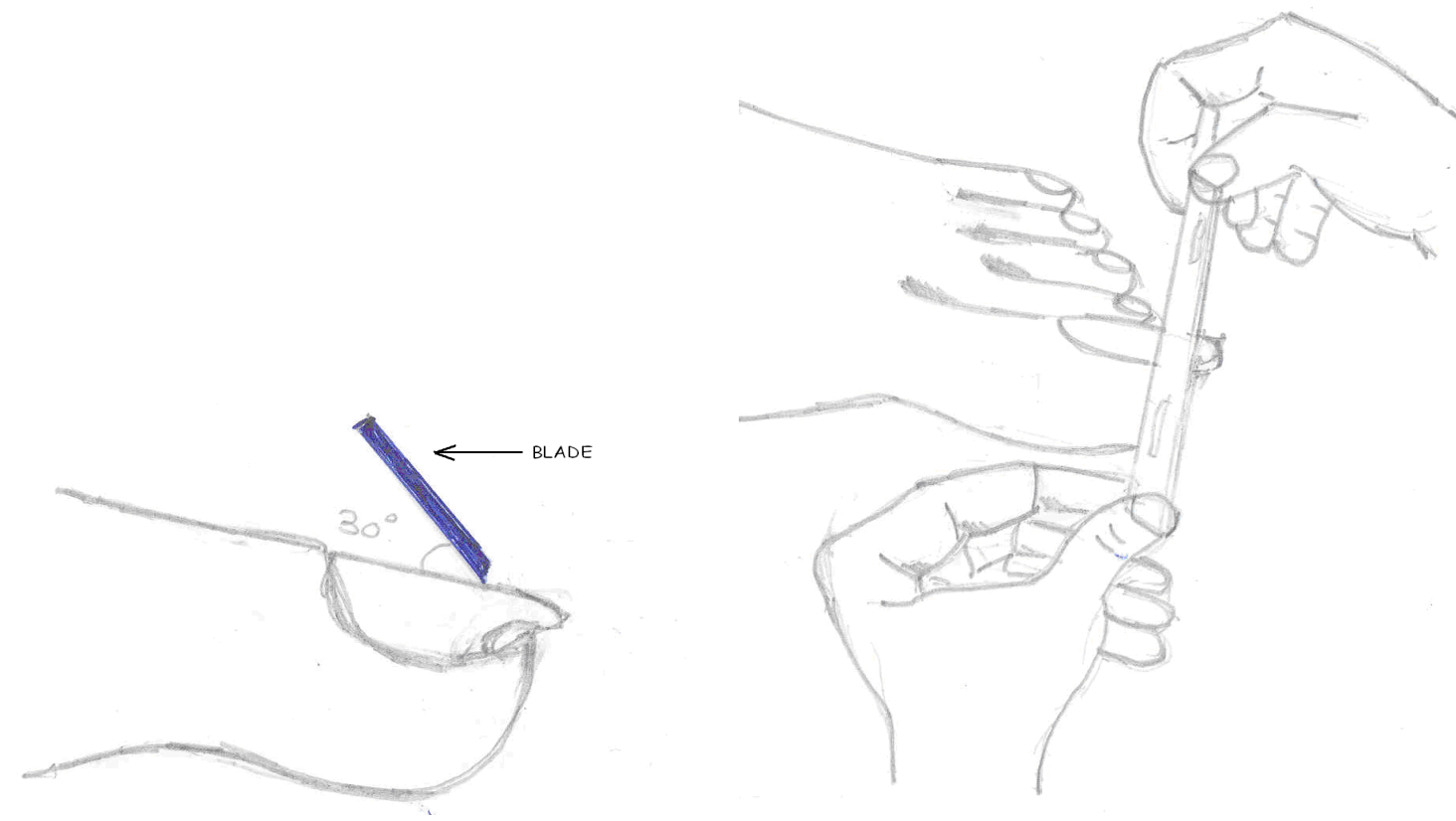

Figure 2- Method of sample collection. 


\section{Results}

\begin{tabular}{|c|c|c|}
\hline Method of collection & Test method positives & Test method negatives \\
\hline Conventional method positives & 14 & 1 \\
\hline Conventional method negatives & 16 & 23 \\
\hline
\end{tabular}

Table 1-Number of positive and negative samples with each test

Of the total 54 samples processed, 31 were positive by either the test or the conventional method. The test method gave 30 positives and the conventional method gave 15 positives. While 16 of the samples that tested positive with the test method were negative according to the conventional method, only one sample that was positive with the conventional method was negative according to the test method (Table 1). The positivity rate for the test method was $96.8 \%$, while the rate for the conventional method was $48.4 \%$.

\section{Discussion}

The disadvantages of taking nail clippings from thick dystrophic nails using the conventional manner are many. Nail clippers are not wide enough to encompass the thickness of the nail and sometimes the procedure causes discomfort to the patient. In addition, the clippings take a long time to clarify in $\mathrm{KOH}$, however small the pieces are. Microscopy too becomes difficult if clarification does not provide a single layer of cells.

In the test method, the technique of obtaining thin nail shavings is easily performed and does not cause any discomfort to the patient. The shavings obtained are extremely thin and do not require prolonged clarification but can be examined almost immediately and give a clear single-celled layer- while maintaining the architecture of the nail so that fungal filaments may be visualized easily.

\section{Conclusions}

The single most important advantage is the higher positivity rate of the test method when compared to the conventional method. In hospitals where fungal culture facilities may not be readily available, reliance on microscopy alone for diagnosis of fungal nail infections is important for management of patients.

\section{References:}

1. Evans EGV, Richardson MD. Medical Mycology a practical approach. Oxford University Press 1989: Page 4.

2. Qureshi HS, Ormsby HA, Kapadia N. Effects of modified sample collection technique on fungal culture yield: nail clipping/scraping versus microdrill. The Journal of the Pakistan Medical Association 2004; 54(6): 301-5.

3. Shemer A, Davidovici B, Grunwald MH, Trau H, Amichai B. Comparative study of nail sampling techniques in onychomycosis. Department of Dermatology, Sheba Medical Center, Tel-Hashomer, Israel 2009. The Journal of Dermatology; 36 (7): 410-4. 